Meta

Journal des tradlucteurs

Translators' Journal

\title{
Petit historique de la publicité française au Canada
}

\section{Marcel Paré}

Volume 17, numéro 1, mars 1972

L’adaptation publicitaire

URI : https://id.erudit.org/iderudit/003786ar

DOI : https://doi.org/10.7202/003786ar

Aller au sommaire du numéro

\section{Éditeur(s)}

Les Presses de l'Université de Montréal

\section{ISSN}

0026-0452 (imprimé)

1492-1421 (numérique)

Découvrir la revue

Citer cet article

Paré, M. (1972). Petit historique de la publicité française au Canada. Meta,

17(1), 52-55. https://doi.org/10.7202/003786ar d'utilisation que vous pouvez consulter en ligne.

https://apropos.erudit.org/fr/usagers/politique-dutilisation/ 


\section{Petit historique de la publicité française au Canada}

Cela ne fait aucun doute, la publicité est l'un des deux plus vieux métiers du monde, l'un servant l'autre et se voyant même parfois attribuer ses comportements. Nous ne remonterons pas jusque-là puisqu'il est bien peu probable qu'on se soit alors servi de la langue française pour vanter sa marchandise.

On a pu croire que les journaux virent le jour avant les annonces et servirent à celles-ci de premier véhicule. Ce serait le contraire qui serait vrai si l'on en croit les biographes du docteur Théophraste Renaudot. Un des premiers à se servir de la publicité de façon méthodique, Renaudot aurait inventé les petites annonces et, au bout de quelques années, en 1631, il aurait, en y ajoutant des nouvelles, transformé en journal sa feuille de réclame. Ce fut la première Gazette française. Jusque-là, la publicité se faisait de vive voix ou de bouche en bouche, au moyen de chansons populaires, des cris des marchands de Paris et des crieurs publics.

Onze ans plus tard, Montréal vit le jour, mais elle dut attendre un siècle et demi sa première gazette, la Gazette du commerce et littéraire, fondée par Fleury Mesplet, imprimeur, avec les conseils (déjà!) de l'Américain Benjamin Franklin. En 1795, la Gazette, journal bilingue, publiait des annonces en anglais et en français. Au bout d'un siècle, ayant peu à peu cessé d'être bilingue, la Gazette devint et reste The Gazette.

En même temps, la publicité française devenait de plus en plus chez nous une publicité traduite, rarement bien traduite, exemple néfaste pour la création française, si tant est qu'il y en eut alors.

Au début du siècle et entre les deux guerres, la production à la chaîne, la grande consommation et l'évolution des techniques publicitaires aux États-Unis donnèrent à la publicité un élan qu'elle n'avait jamais connu, qui s'accentua avec les efforts de propagande de la Première Grande Guerre, fit un bond énorme avec l'avènement des nouveaux véhicules de diffusion, la radio et, plus tard, la télévision, puis avec les raffinements de l'électricité, de la photographie, de l'imprimerie, etc. 
La publicité devenait une puissante technique, au service d'un art à la fois subtil et grossier, qui semblait, et semble encore aujourd'hui, vouloir imposer comme telle à tout l'univers sa nationalité américaine.

La petite clientèle de quelque cinq millions de francophones perdus dans l'Amérique anglophone occupait bien peu de place dans les préoccupations publicitaires du temps. Les publicitaires de langue française étaient rares et les quelques traducteurs qui possédaient la formation ou les connaissances voulues ne se trouvaient pas surtout dans les agences de publicité mais principalement au Gouvernement fédéral et dans quelques journaux et stations de radio où ils devaient alors tout traduire : dépêches, communiqués, annonces et même les notices accompagnant des disques comme ceux de Maurice Chevalier.

Les agences de publicité américaines ou les fabricants de produits et articles mis sur le marché québécois, lorsqu'ils ne confiaient pas la traduction de leur réclame au premier venu, en chargeaient les journaux et stations de radio avec lesquels ils faisaient affaire. Comme ils faisaient à ceux-ci la faveur (sic) d'acheter leur temps ou leurs pages, ils en attendaient la faveur d'une traduction gratuite. Évidemment, la même annonce anglaise se retrouvait en autant de versions françaises qu'elle avait eu de traducteurs. On s'étonnait ensuite de constater qu'elles ne semblaient pas toutes avoir dit la même chose.

La Seconde Grande Guerre fit faire un nouveau bond à la publicité et déclencha en même temps certains mécanismes nouveaux au sein des moyens de diffusion de langue française.

En effet, quelques représentants de journaux et de stations de radio canadiensfrançais, qui faisaient partie de certains organismes de publicité mis sur pied par le gouvernement durant la guerre, exigèrent et obtinrent de faire adapter et, un peu plus tard, de créer, en français, des annonces et même des campagnes publicitaires. Ceci eut pour résultat d'amener quelques jeunes Canadiens français à la publicité et aux techniques publicitaires.

Cette première et timide prise de conscience s'accentua durant la période de prospérité de l'après-guerre. Les quelques publicitaires canadiens-français d'avant la guerre, on les comptait sur les doigts de la main avant 1939, se sont peu à peu multipliés. Ils sont venus de la radio et des journaux principalement. La radio avait été la première à offrir un peu plus de liberté aux traducteurs ou aux rédacteurs car elle n'imposait pas les contraintes matérielles, d'ordre graphique surtout, de l'annonce imprimée : image, mise en page, espace; à la radio, le traducteur ne se sentait pas autant l'esclave du texte original anglais.

La réclame radiophonique eut la liberté d'acquérir relativement plus de personnalité et de fraîcheur que celle des autres véhicules publicitaires. Si bien que ce que les agences appelaient leur service français était alors, le plus souvent, le service de rédaction et de réalisation des annonces destinées à la radio. C'est le personnel des stations de radio du Québec qui a peut-être contribué le plus à la formation de ces services dans les agences. Il n'en reste pas moins qu'en général, les journaux se sont rarement souciés de corriger les mauvaises habitudes de leurs clients, quelle qu'ait été leur langue ; la radio non plus, sauf la société Radio-Canada qui eut une influence, dans l'ensemble, fort heureuse pour la 
publicité, mais très diversement appréciée par ceux qui en ont été ou les bénéficiaires ou les soi-disant victimes.

Mais l'évolution continuait et les jeunes se préparaient, même en l'absence d'une école spécialisée, à une carrière dans la publicité ou dans les domaines connexes de l'activité commerciale. Quelques-uns sont parvenus à des postes de direction mais sans toujours pouvoir, pour autant, obtenir une plus grande mesure de liberté dans la préparation de la publicité de langue française. À ces nouveaux venus, on permettait tout au plus de veiller à ce que la version française créée par l'agence ne pèche pas trop contre les règles du bon goût ou de la grammaire, ou ne heurte pas trop de front les traditions, les habitudes et les sentiments des Canadiens français.

D'autres se sont lancés courageusement dans la mêlée pour concurrencer les agences déjà en place et qui, sauf deux ou trois exceptions, appartenaient toutes à des intérêts anglo-canadiens ou américains. Ils ont fait de louables efforts pour établir ce qu'on aurait appelé une publicité française au Canada. $\mathrm{Si}$, à travers d'authentiques succès qui témoignent de leur compétence, ils n'ont pas toujours atteint tous les objectifs visés, la faute n'en est pas à leur manque d'expérience ou de talent, elle tient plutôt à la difficulté de convaincre le tout-puissant distributeur des biens de consommation, et surtout ses agences de publicité, tant au Canada qu'aux États-Unis, qu'il pourrait trouver intérêt à confier sa publicité française dans le Québec à une équipe québécoise de langue française qui pourrait créer des campagnes mieux adaptées au marché visé.

Nous avons passé l'époque où le publicitaire canadien-français était le plus souvent l'homme-à-tout-faire-et-surtout-correcteur-d'épreuves de l'agence. Nous avons aujourd'hui des spécialistes de la rédaction ou de la commercialisation, de la communication ou de la distribution, de la réalisation ou de la présentation. Ils ont la compétence voulue pour bien faire ce qu'on attend d'eux. Cela n'empêche pas que ceux qui se sentent le plus frustrés parmi eux, ce sont les rédacteurs et les traducteurs. Devant les acrobaties terminologiques apparemment faciles de l'anglais, ils désespèrent de trouver l'équivalent français et c'est, pour la plupart, le vocabulaire qui reste la principale pierre d'achoppement, soit parce qu'ils le connaissent mal, soit que même ceux qui le connaissent bien refusent parfois de l'utiliser correctement, croyant à tort que les lecteurs ou les auditeurs auxquels ils s'adressent ne comprendront pas.

Si le mot boisson peut encore parfois prêter chez nous à équivoque en raison du sens particulier qu'il a conservé au Québec et qui peut parfois en rendre le maniement ambigu, on devrait quand même s'efforcer de l'employer à la place du mot breuvage. Sur les menus de restaurant, entre autres, il n'y a aucune équivoque possible lorsqu'on y énumère tout ce qu'on offre sous le nom de boisson.

Par ailleurs, pour garder le même exemple, le mot breuvage est un mot français qui a déjà eu le sens qu'on lui donne encore chez nous, comme tant d'autres de nos mots dont le sens resté immuable ici a connu, dans son pays d'origine, des glissements qui l'ont rendu désuet dans le sens où nous l'employons. 
Il y a des erreurs bien plus importantes à condamner que cet emploi attardé de breuvage.

N'a-t-on pas pratiquement réussi à implanter le mot cuisinière pour désigner cet appareil électroménager qui ne sert plus aujourd'hui qu'à faire la cuisine ?

Si la publicité réussit à imposer des noms hier inconnus comme Nylon, Cellophane, Frigidaire, et même des termes en langue étrangère, comme Head \& Shoulders, véritablement difficiles à prononcer et à retenir pour des gens qui ne parlent pas habituellement cette langue, je ne vois pas pourquoi elle ne pourrait pas tout aussi bien faire accepter, grâce à son omniprésence et à son autorité, des mots français que tout le monde aura tôt fait de comprendre.

Quant à la grammaire, je ne crois pas que le fait d'en ignorer les règles puisse en quoi que ce soit apporter quelque bénéfice à la publicité. Au contraire. C'est le respect des règles qui va permettre à la langue de véhiculer convenablement les pensées qu'on lui confie. La langue devient une messagère suspecte le jour où on veut la libérer des règles qui la régissent. Il n'en résulte alors que confusion, incompréhension et, dans le cas de la publicité, des ennuis économiques à longue ou à brève échéance.

Il faut ajouter que ce n'est pas toujours la publicité traduite qui pèche le plus sérieusement contre la langue, c'est souvent la publicité écrite par des francophones, hélas! Si les mauvais exemples qu'ils ont eus sous les yeux pendant longtemps en sont l'explication, ils ne servent quand même pas d'excuse.

Enfin, la prise de conscience toute récente des francophones à l'égard de leur langue a aussi exercé une heureuse influence sur la publicité française au Canada. On se rend compte que depuis quelques années la publicité française se cherche une personnalité canadienne.

Elle a conquis une place de plus en plus importante, mais elle n'est pas encore arrivée là où il faudrait. Le nombre et la compétence accrue de ceux qui font aujourd'hui carrière en ce domaine lui permettront de faire d'autres progrès pourvu qu'on puisse désormais assurer une relève de qualité. Comme bien d'autres, je crois que l'enseignement de la publicité et de toutes les techniques qui constituent les éléments de cette profession est désormais indispensable chez nous pour assurer cette relève. Bien sûr, nous aurons à puiser dans l'expérience américaine, mais pourquoi n'en ferions-nous pas autant pour l'expérience européenne, qu'elle soit française, italienne ou anglaise, qui a aussi gagné ses épaulettes. Nous sommes nord-américains, il n'est pas permis d'en douter, mais notre originalité en publicité reposera en grande partie sur notre habileté à assimiler non seulement les techniques de nos voisins du sud mais d'autres aussi qui n'ont pas moins de valeur. Il ne faut cependant pas oublier que la langue que nous parlons et que nos publicitaires sont chargés d'écrire est le français et ce n'est pas en le dégradant que notre publicité acquerra une personnalité canadienne. Ce serait dommage, car cela finirait par nous couper à tout jamais du monde francophone et des richesses qu'il représente pour nous, quoi qu'on en dise.

Marcel Paré 